\title{
Controls on the magnitude-frequency scaling of an inventory of secular landslides
}

\author{
M. D. Hurst, M. A. Ellis, K. R. Royse, K. A. Lee, and K. Freeborough \\ British Geological Survey, Keyworth, Nottingham, NG12 5GG, UK \\ Correspondence to: M. D. Hurst (mhurst@bgs.ac.uk) \\ Received: 14 June 2013 - Published in Earth Surf. Dynam. Discuss.: 1 July 2013 \\ Revised: 6 November 2013 - Accepted: 15 November 2013 - Published: 11 December 2013
}

\begin{abstract}
Linking landslide size and frequency is important at both human and geological timescales for quantifying both landslide hazards and the effectiveness of landslides in the removal of sediment from evolving landscapes. The statistical behaviour of the magnitude-frequency of landslide inventories is usually compiled following a particular triggering event such as an earthquake or storm, and their statistical behaviour is often characterised by a power-law relationship with a small landslide rollover. The occurrence of landslides is expected to be influenced by the material properties of rock and/or regolith in which failure occurs. Here we explore the statistical behaviour and the controls of a secular landslide inventory (SLI) (i.e. events occurring over an indefinite geological time period) consisting of mapped landslide deposits and their underlying lithology (bedrock or superficial) across the United Kingdom. The magnitude-frequency distribution of this secular inventory exhibits an inflected power-law relationship, well approximated by either an inverse gamma or double Pareto model. The scaling exponent for the power-law scaling of medium to large landslides is $\alpha=-1.71 \pm 0.02$. The small-event rollover occurs at a significantly higher magnitude $\left(1.0-7.0 \times 10^{-3} \mathrm{~km}^{2}\right)$ than observed in single-event landslide records $\left(\sim 4 \times 10^{-3} \mathrm{~km}^{2}\right)$. We interpret this as evidence of landscape annealing, from which we infer that the SLI underestimates the frequency of small landslides. This is supported by a subset of data where a complete landslide inventory was recently mapped. Large landslides also appear to be under-represented relative to model predictions. There are several possible reasons for this, including an incomplete data set, an incomplete landscape (i.e. relatively steep slopes are under-represented), and/or temporal transience in landslide activity during emergence from the last glacial maximum toward a generally more stable late-Holocene state. The proposed process of landscape annealing and the possibility of a transient hillslope response have the consequence that it is not possible to use the statistical properties of the current SLI database to rigorously constrain probabilities of future landslides in the UK.
\end{abstract}

\section{Introduction}

This paper describes the generation and analysis of a secular landslide inventory (SLI) derived from the UK National Landslide Database (NLD) (Foster et al., 2012). We tackle two basic questions. First, does this secular landslide inventory reflect similar or different statistical properties as generally better constrained, single-event driven inventories and local-scale historical inventories (cf. Van Den Eeckhaut et al., 2007)? Second, what role is played by the underlying lithology and type of landslide in controlling the statistical properties of the inventory? The drivers for the current analysis include the need to quantify landslide hazards and to better understand erosional processes in long-term landscape evolution.

Landslides pose a significant hazard to human life and infrastructure. In the US, Japan, Italy and India landslides have been estimated to result in economic losses for each in excess of (1990 USD) USD 1.0 billion per annum (Schuster, 1996). Between 2004 and 2010 there were at least 2600 fatal landslides globally, with 32000 associated fatalities (Petley, 2012). Whilst loss of life due to landsliding in the UK is relatively rare, landslides pose a risk to infrastructure and are 
relevant in land use planning (Gibson et al., 2013). Landslides also have the potential to disrupt transport links (Winter et al., 2010), and land use change has been acknowledged to influence the occurrence of landslides throughout the world (Glade, 2003). Given that landslide behaviour is in part dictated by levels and frequency characteristics of precipitation, there is concern that the patterns and severity of landsliding may be affected by future climate change (Crozier, 2010; Keiler et al., 2010; Korup et al., 2012).

Landslides are important geomorphic processes which generate and transport significant volumes of rock, regolith and soil (e.g. Korup et al., 2010; Larsen et al., 2010). Landslides occur in a variety of styles, dictated by a web of interrelated factors, including material properties (e.g. soil type and thickness, bedrock type, the orientation and spacing of discontinuities), landscape morphology (e.g. slope, topographic convergence, aspect) and climate (e.g. freeze-thaw and shrink-swell cyclicity, pore-water pressures). Whilst large landslides are often perceived to be the most hazardous, small landslides occur most frequently; therefore quantifying the size-frequency distribution for landslide events is important to the assessment of landslide hazard and to land use planning (Malamud et al., 2004). Landslides may also be a significant component of the sediment budget in a landscape and hence understanding their size-frequency characteristics is important to studies of long-term landscape evolution (Stark and Guzzetti, 2009). In a recent review, Guzzetti et al. (2012) recognised that detailed inventories of landslides are lacking, advocating them as a vital tool in assessing susceptibility and risk at a variety of time and length scales. Inventories may focus at a variety of temporal and spatial scales, from a single drainage basin (Guzzetti et al., 2008) to national scale (Trigila et al., 2010; Van Den Eeckhaut and Hervás, 2012); from single event-triggered landslide clusters (Parker et al., 2011) to multi-temporal historical records (Galli et al., 2008) with unconstrained landslide ages.

Several studies have proposed that the non-cumulative size-frequency distribution for landslides (i.e. the number of slides of a give size occurring over a given length of time or within a given area) follows a negative power-law relationship for medium to large landslides (e.g. Guzzetti et al., 2002; Hovius et al., 1997; Pelletier et al., 1997; Stark and Hovius, 2001; Turcotte et al., 2002). Estimates of the exponent $\alpha$ for power-law scaling of large events vary from $\alpha=1.4$ up to $\alpha=3.3$ (Van Den Eeckhaut et al., 2007). Van Den Eeckhaut et al. (2007) report an average value of $\alpha=2.3 \pm 0.6$ based on a compilation of inventories, and Malamud et al. (2004) suggested $\alpha=2.4$ might be universally applicable to eventtriggered inventories based on consensus between three contrasting event-driven data sets. Larsen et al. (2010) caution that estimates of volume of material transported by landslides may be very sensitive to this scaling exponent, resulting in prediction errors of over an order of magnitude. The scaling exponents may vary with underlying geology (e.g. Frattini and Crosta, 2013; Guzzetti et al., 2008), and the type of fail- ure event (e.g. Brunetti et al., 2009; by analysis of landslide volume rather than area statistics).

A negative power-law model only holds for landslides larger than a particular size, and this minimum size will vary between different inventories. Landslide size-frequency distributions from around the world consistently exhibit a rollover to a positive relationship for smaller landslides, with the size of landslide at which the rollover occurs varying between inventories (e.g. Brardinoni and Church, 2004; Guzzetti et al., 2008; Malamud et al., 2004; Van Den Eeckhaut et al., 2007). For complete landslide inventories the rollover has been interpreted as resulting from the interplay of cohesion and friction, whereby these forces offer resistance to landsliding for small and large landslides respectively (Guzzetti et al., 2002; Malamud et al., 2004; Pelletier et al., 1997; Stark and Guzzetti, 2009). Alternatively (or perhaps additionally), the rollover has been attributed to the under-sampling of small landslides when compiling the inventory. Under-sampling might occur due to evidence of small landslides being rapidly removed through erosion, the reworking of deposits and recolonisation by vegetation (Brardinoni and Church, 2004), difficulties in identification of smaller landslides, or resolution issues with remotely sensed data sets (Malamud et al., 2004; Stark and Hovius, 2001). The rollover occurs at larger landslide sizes in historical inventories where evidence of smaller landslides has been lost from the geomorphic record (Malamud et al., 2004; Van Den Eeckhaut et al., 2007). Additionally Van Den Eeckhaut et al. (2007) demonstrated that a historical inventory of the Flemish Ardennes in Belgium was well characterised by the superposition of two power laws, one characterising the sizefrequency of large, old landslides driven by natural processes and another for recent landslide activity influenced by human impacts on the landscape. Two statistical distributions have been proposed to model the rollover in size-frequency distributions of terrestrial landslides. Stark and Hovius (2001) found landslide inventories from New Zealand and Taiwan could be fit by a double Pareto distribution. Malamud et al. (2004) favour fitting an inverse gamma function, which can also account for the rollover. An inverse gamma function provided a good approximation of the size frequency distribution of data sets from Italy, Guatemala and the USA, with different trigger conditions (snowmelt, storm and earthquake triggers respectively) (Malamud et al., 2004). The three inventories were considered to be complete (i.e. the rollover is real and not a result of under-sampling of small landslides) thereby leading Malamud et al. (2004) to suggest the model as a general fit for any complete event-driven landslide inventory.

The universality of such a general model for landslide distributions has not been verified. Malamud et al. (2004) suggest it has applicability to historic, multi-trigger inventories since the model can be fitted to the large landslide tail of a historical inventory which is more likely to be a substantially complete record, since evidence of larger landslides 
will persist for longer time periods in a landscape. As a result, by comparison to the proposed general distribution, the total number of landslides missing from an inventory can be predicted even for an incomplete landslide inventory (Malamud et al., 2004). Historic inventories (e.g. Guzzetti et al., 2008; Trigila et al., 2010) show similar power-law scaling with $\alpha \approx 2.4$ but with the location of the rollover offset towards larger landslides. Guzzetti et al. (2008) interpret the offset as due to difficulty in documenting smaller landslides from aerial photos and their tendency to amalgamate (i.e. incompleteness of the record). The difference might also relate to the loss of smaller landslides from the record due to landscape annealing by erosion, reworking of deposits and recolonisation by vegetation (Brardinoni and Church, 2004).

The concept of a general model for a landslide sizefrequency relationship may seem at odds with the range of factors expected to influence landslide occurrence, such as climate, vegetation, material properties of bedrock/regolith and the type/style of failure. Clarke and Burbank (2010) compared the size-frequency distribution of two landslide inventories in Fiordland and the Southern Alps in New Zealand, which are dominated by igneous and highgrade metamorphic lithologies, and low-grade metamorphic lithologies respectively. Whilst power-law scaling exponents were similar between the two sites $(\alpha \approx 1.07$ and 1.16, respectively), the sizes of the largest landslides were roughly an order of magnitude larger, and the position of the rollover in frequency was also shifted toward larger landslides in the Southern Alps compared to Fiordland. Frattini and Crosta (2013) constructed synthetic size-frequency distributions using slope stability analysis to suggest that less resistant materials tend to promote more shallow landslides whilst more resistant lithologies tend toward deeper landslides with limited numbers of smaller landslides. This has important implications for the volume of materials transported by landslides in different materials. Larsen et al. (2010) compiled a global data set of landslide geometries and observed that scaling of volume with area for shallow, soil landslides has a lower exponent than for deep-seated bedrock landslides. A general model for the distribution of landslides (Malamud et al., 2004) may not take into account lithologic variability and differences in the type of mass movement processes (which are likely linked themselves).

In this study, we attempt to quantify and explain the statistical properties of a national-scale secular landslide inventory, test the geomorphic completeness (i.e. degree of landscape annealing) of such an inventory and estimate the number of landslides that might be missing from the geomorphic record. We link the frequency distribution of landslide sizes to the lithology or deposits in which they occur to assess whether particular lithologies may have been more prone to landsliding. Finally, we select landslides in which the type of failure was known in order to assess whether scaling relationships are a function of landslide type. We achieve this by generating a national-scale SLI from the NLD in the
United Kingdom, which we combine with mapped landslide deposits, and maps of underlying geology.

\section{Data and methods}

\subsection{Landslide data}

The NLD is an extensive inventory of ancient and recent landslides in the UK (Fig. 1) (Foster et al., 2012). The database is managed by the British Geological Survey (BGS), having inherited and expanded a database initially compiled from a desk study carried out by Geomorphological Services Limited in the late 1980s to document the occurrence of landslides in the UK on behalf of the UK Government's Department of the Environment (Jones and Lee, 1994). The database consisted of records compiled from journal articles and reports, and maps and reports held by the BGS (Foster et al., 2012). The NLD has expanded from this origin and is maintained and managed by the BGS. The database now comprises a series of points $(n=16808$; November 2012) recording the location of known landslides, the precise timing of which is often unknown. Many of these points have been through a quality assurance (QA) procedure ( $n=13108$; November 2012) verifying their location by reference to previous studies, maps or field surveys.

The NLD includes a detailed record of many attributes of a particular landslide event including landslide type, slide material, presence of vegetation, hillslope gradient and estimated age (see Foster et al., 2012, for further details), but the availability of these data depend on when and by whom the individual landslide was recorded. During QA the points are related to mapped landslide deposits recorded by geological mapping at $1: 10000$ and $1: 50000$ scales over the last century by the BGS (DiGMap; British Geological Survey, 2009, 2010). These mapped deposits have been recorded by a multitude of geologists whose primary concern may not have been the precise recording of landslide deposits, thus the quality of the data set is likely to be highly variable. The NLD is not considered a complete inventory of landslide occurrence in the UK. The data were collated from a multitude of sources and therefore approaches to mapping are unlikely to have been consistent. Additionally the spatial coverage of landslide mapping is unlikely to have been consistent. As part of the continuing collection, updating and verification of landslide information by the BGS, existing landslide data are subjected to a standardised QA procedure designed to improve the consistency and reliability of the these data sets, and areas with poor coverage are identified for detailed resurveying (Foster et al., 2012). These activities are vital to planning and development within the UK (Foster et al., 2012), and is a fundamental component in the nationwide assessment of landslide susceptibility (Walsby, 2008). 


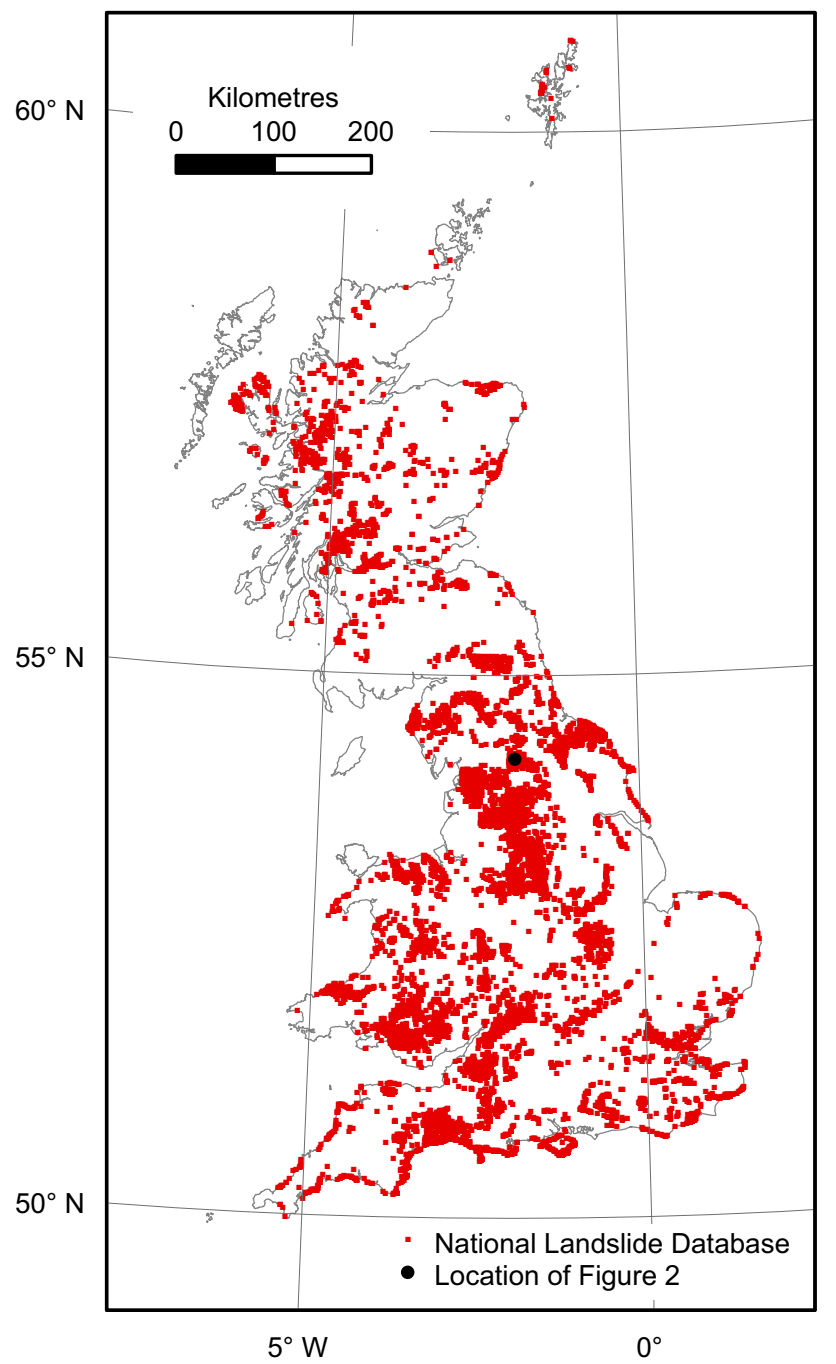

Figure 1. UK Map showing the distribution of landslide points in the NLD which have undergone quality assurance control at the British Geological Survey. Black circle shows the location of Fig. 2.

\subsection{Sampling methods}

The magnitude-frequency relationship for landslides in the UK was quantified based on linking quality assured landslides in the NLD (Fig. 1) to their associated mapped landslide deposits (for either 1:10000 and 1:50000 scale maps), where available, using GIS software. We emphasise that in this contribution we are analysing the deposit area rather than the total failure plus run-out area as is more commonly reported (Malamud et al., 2004), because only landslide deposit areas have been recorded as part of BGS's geological mapping.

During the quality assurance procedure, if a landslide reported in the NLD can be allied to a mapped landslide deposit, the coordinates of the point record in the NLD are moved to the location of highest elevation at the edge of the mapped landslide polygon. In some cases where the head scarp of the landslide is visible in aerial photographs or topographic data, the point coordinates in the NLD will alternatively be moved to the highest point on the observed scarp. In order to link points in the NLD to mapped landslide polygons we used ArcGIS software to measure the shortest distance between records in the NLD and their nearest deposit area polygon. Where this distance was less than $50 \mathrm{~m}$ we considered that the point and polygon were related and hence attributes of the mapped landslide deposit polygons were linked to the NLD (e.g. Fig. 2; box 2) to generate the sample used in the current analysis.

There were a number of caveats to this linking procedure requiring consideration. Firstly, mapped deposits may consist of the amalgamation of several proximal landslide runouts, or be the result of landslide reactivation and therefore have multiple associated events in the NLD (e.g. Fig. 2; box 1). In order to isolate individual event deposits, we omitted records where multiple points from the NLD were associated with a single landslide deposit polygon $(n=1944)$. Similarly, we omitted occurrences of a landslide deposit polygon that had no associated nearby $(<50 \mathrm{~m})$ records in the NLD $(n=1177)$. We also omitted records in the NLD with no associated mapped landslide deposit (Fig. 2; red points, $n=6026$ ). Finally, we also omitted coastal landslides (via a $500 \mathrm{~m}$ buffer from the UK coastline) in order to restrict our analysis to strictly terrestrial landslides $(n=386)$. The resulting sampled data set consists of 8452 single landslide eventdeposit area pairs. We subsequently refer to this filtered landslide data set to as the SLI.

To quantify landslide size, we used ArcGIS to measure the aerial extent of each mapped landslide deposit polygon retained in the SLI. We used the centroid points of mapped deposit polygons to sample the underlying lithology and the presence/absence of superficial material from digital geological maps (DiGMap; British Geological Survey, 2009, 2010), following the BGS's standardised rock classification scheme (RCS) (Styles et al., 2006). The geology of the United Kingdom is quite diverse, with over 180 separate RCS codes identified during sampling. In order to look for lithologic control on landslides, we split these into seven broad lithologic groups: superficial deposits, mudstones, interbedded sedimentary units, coarse clastic sedimentary units (sandstones and coarser), carbonates, metamorphics and igneous (Table 1). Superficial deposits refer to young (Quaternary age) geological deposits (glacial or alluvial) which rest on bedrock (we are not able to distinguish soil from bedrock landslides). Interbedded units refer to sequences where fine grained clastics (i.e. mudstones) are interbedded with coarser, usually more resistant layers. 
Table 1. Parameter estimates of power-law model for the entire SLI data set and subsets grouped by lithology. Power laws were fitted following Clauset et al. (2009) with error ranges reported as standard errors.

\begin{tabular}{llc}
\hline Data set & No. Landslides & $\alpha$ \\
\hline SLI & 8453 & $1.71 \pm 0.01$ \\
Superficial & 2497 & $1.82 \pm 0.03$ \\
Mudstone & 2339 & $1.69 \pm 0.02$ \\
Interbedded & 1986 & $1.71 \pm 0.02$ \\
Clastic & 1188 & $1.67 \pm 0.03$ \\
Carbonate & 268 & $1.58 \pm 0.04$ \\
Metamorphic & 111 & $1.53 \pm 0.05$ \\
Igneous & 64 & $1.82 \pm 0.12$ \\
\hline
\end{tabular}

\subsection{Statistical analysis}

\subsubsection{Quantifying landslide size distributions}

The non-cumulative frequency density $\left(F_{\mathrm{D}}\right)$ of a landslide inventory is given by the number of landslides $\mathrm{d} N$ over the range of areas $\mathrm{d} A$. Probability density $\left(P_{\mathrm{D}}\right)$ can be estimated for a landslide data set as $F_{\mathrm{D}}$ normalised to the total number of landslides in the inventory $N_{\mathrm{T}}$ according to

$P_{\mathrm{D}}=\frac{1}{N_{\mathrm{T}}} F_{\mathrm{D}}=\frac{1}{N_{\mathrm{T}}} \frac{\mathrm{d} N}{\mathrm{~d} A}$,

in which $P_{\mathrm{D}}$ is the probability of a landslide with area $A\left[\mathrm{~L}^{2}\right]$ (Malamud et al., 2004). We calculated $F_{\mathrm{D}}$ and $P_{\mathrm{D}}$ for the SLI data set by sorting the data into bins spaced evenly in $A$ in logarithmic space.

\subsubsection{Models for landslide size distribution}

As previously noted, the scaling of probability density $P_{\mathrm{D}}$ with landslide size for medium-large landslides can be described by a power law:

$P_{\mathrm{D}}=\frac{\alpha-1}{A_{\min }}\left(\frac{A}{A_{\min }}\right)^{-\alpha}$,

where $A_{\min }$ is the landslide size cutoff for power-law scaling and $\alpha$ is a dimensionless scaling exponent. Stark and Hovius (2001) proposed using a double Pareto model to describe the size distribution of observed landslides, which accounts for under-sampling of smaller landslides. In the model, $P_{\mathrm{D}}$ is a function of two scaling exponents, $\alpha_{\mathrm{p}}$ and $\beta$, which describe the rate of decay for large and small landslides respectively, either side of a peak landslide area $A_{\text {peak }}$ :

$P_{\mathrm{D}}=\frac{\beta}{A_{\text {peak }}} \times \frac{\left[1+\left(\frac{A_{\max }}{A_{\text {peak }}}\right)^{-\alpha_{\mathrm{p}}}\right]^{\frac{\beta}{\alpha_{\mathrm{p}}}}}{\left[1+\left(\frac{A_{\max }}{A_{\text {peak }}}\right)^{-\alpha_{\mathrm{p}}}\right]^{1+\frac{\beta}{\alpha_{\mathrm{p}}}}} \times\left(\frac{A}{A_{\text {peak }}}\right)^{-\left(\alpha_{\mathrm{p}}+1\right)}$,

where $A$ is landslide area, $A_{\max }$ is the largest landslide in the data set, $A_{\text {peak }}$ is the area at which the rollover occurs,

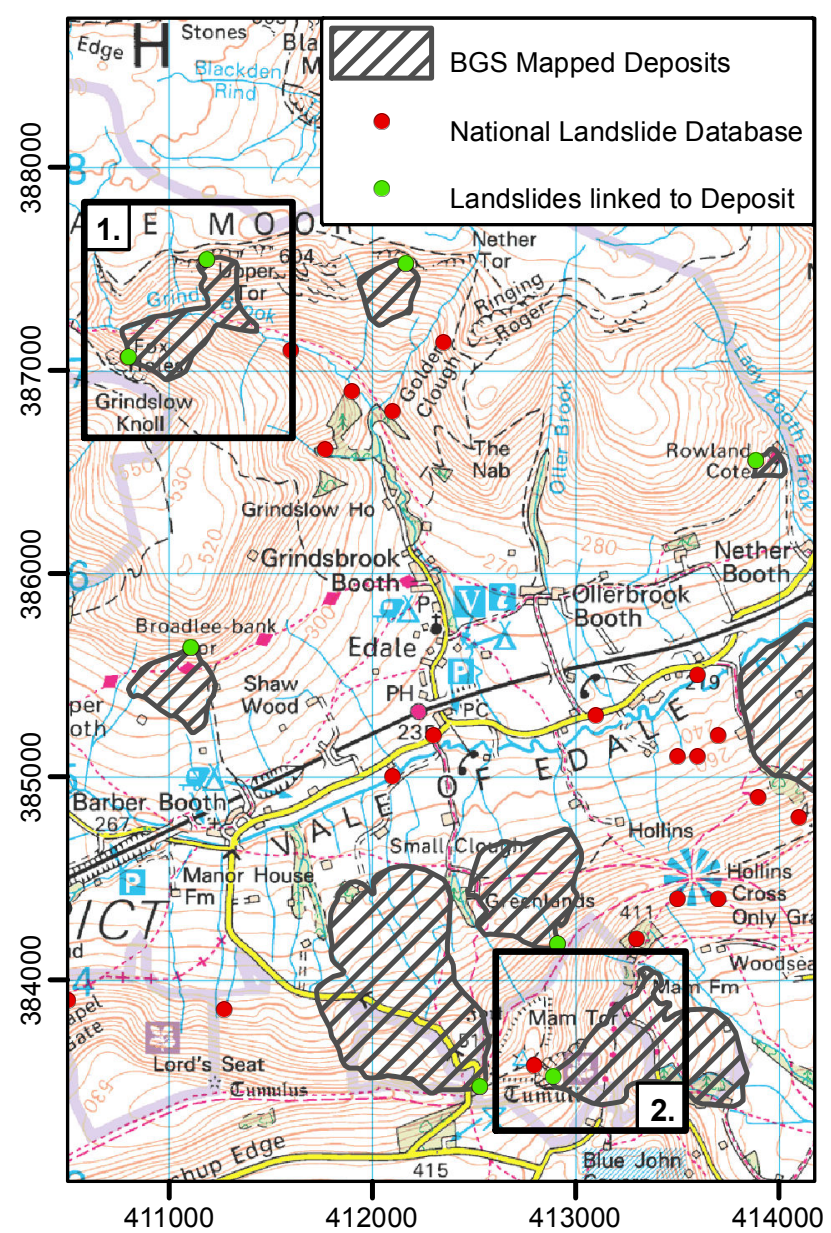

Figure 2. Example map from the Vale of Edale in Derbyshire, showing the locations of points in the NLD (red), and mapped landslide deposits (hatched). Here, there were significantly more events in the database than there were polygons of mapped deposits, probably due to the scale at which mapping took place (1:50 000). Landslide events (red) with no associated, mapped deposit were removed from subsequent analysis. Box 1 highlights a scenario where a single mapped deposit polygon is a composite of two separate landslide events; hence these data are not included in later analysis. Box 2 shows an occasion where a landslide event (red), placed on the back scarp during QA, has been associated with a nearby polygon and linked (green). The spatial reference system is British National Grid; the units are metres. OS topography (C) Crown Copyright. All rights reserved. 100017897/2010.

$\alpha_{\mathrm{p}}$ is the exponent controlling negative power-law scaling for $A_{\text {peak }}<A<A_{\max }$, and $\beta$ is the exponent controlling positive power-law scaling when $0<A<A_{\text {peak }}$ (Stark and Hovius, 2001). Note that the negative power law scaling $\alpha$ in Eq. (2) is equivalent to $\alpha_{\mathrm{p}}+1$. Similarly, Malamud et al. (2004) modelled the probability density of a landslide inventory with a three-parameter inverse gamma function, which acts as an inverse power law for medium-large landslides: 


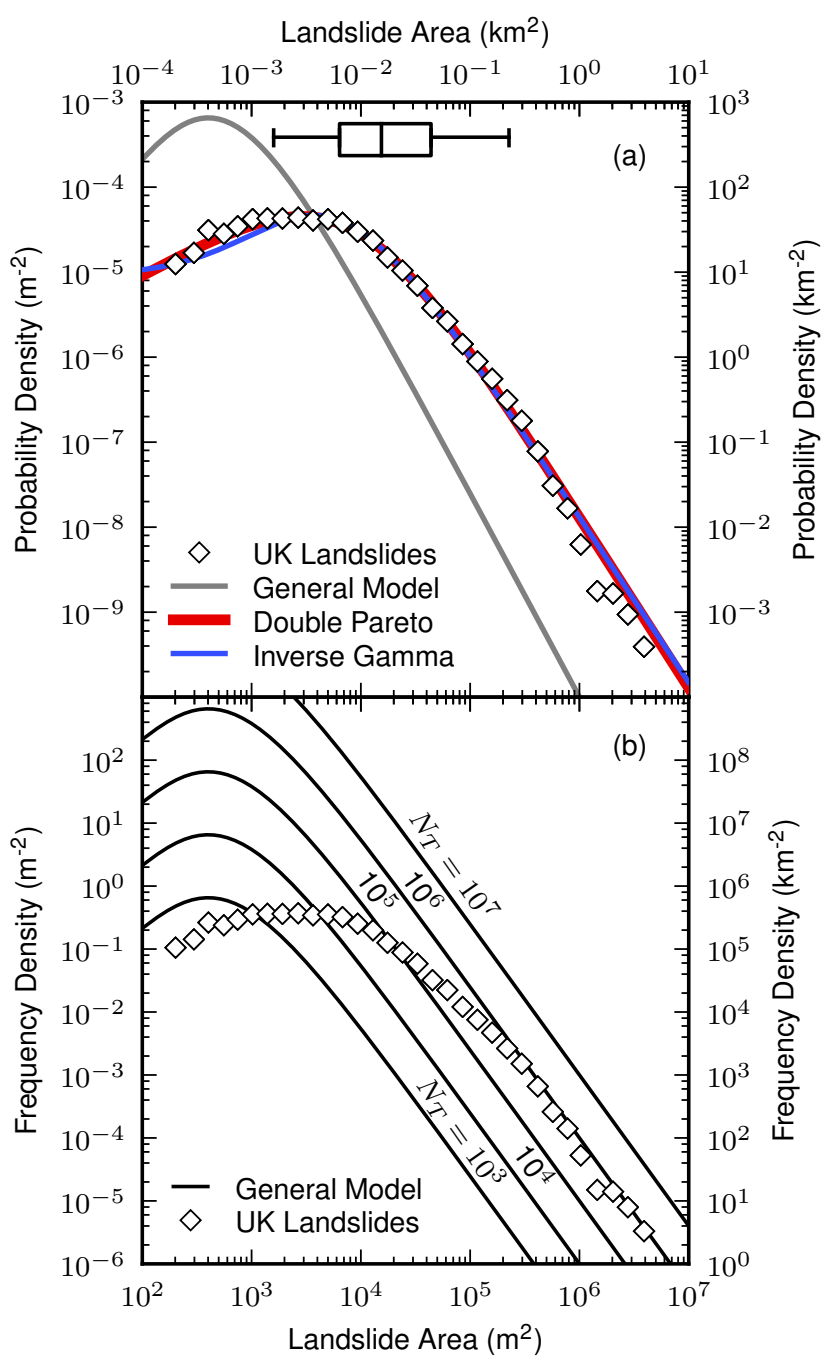

Figure 3. (a) Probability distribution of landslide deposit area for $n=8453$ landslides in the UK organised into bins spaced evenly in logarithmic space (open diamonds). Solid red and blue lines show MLE of a double Pareto function $\left(\alpha_{\mathrm{p}}=1.01 \pm 0.01 ; \beta=1.71 \pm 0.07\right.$; $\left.A_{\text {peak }}=8.09 \pm 0.6 \times 10^{-3} \mathrm{~km}^{2}\right)$ and inverse gamma function $\left(\alpha_{g}=\right.$ $\left.0.95 \pm 0.02 ; r=10.9 \pm 0.4 \times 10^{-3} \mathrm{~km}^{2} ; s=-1.91 \pm 0.08 \times 10^{-3} \mathrm{~km}^{2}\right)$, respectively (error ranges based on one standard deviation of bootstrapped MLE parameters). The grey line is a proposed general distribution for landslides put forward by Malamud et al. (2004). Box plot shows the median (central line), upper/lower quartiles (extent of rectangle) and 5th and 95th percentiles (whiskers) of area data with a median value of $1.53 \times 10^{-2} \mathrm{~km}^{2}$. (b) Frequency density distribution for landslides in the UK. Solid lines represent the general distribution proposed by Malamud et al. (2004) for varying total number of landslides $N_{\mathrm{T}}$.

$P_{\mathrm{D}}=\frac{1}{r \Gamma\left(\alpha_{g}\right)}\left(\frac{r}{A-s}\right)^{\alpha_{g}+1} \exp \left(\frac{r}{A-s}\right)$,

where $\alpha_{g}$ is the exponent setting the inverse power-law scaling for large landslides (again note that $\alpha$ is equivalent to $\left.\alpha_{g}+1\right), r\left[\mathrm{~L}^{2}\right]$ is a parameter controlling the location of the peak in the probability distribution and $s\left[\mathrm{~L}^{2}\right]$ controls the rate of decay for small landslide areas.

\subsubsection{Statistical analysis}

To visualise the size distribution of the SLI we calculated $F_{\mathrm{D}}$ and $P_{\mathrm{D}}$ following Sect. 2.3.1 for the data set as a whole and for subsets grouped by lithology and landslide type (see Figs. 3, and 4). We use a maximum likelihood estimation (MLE) approach to fit statistical models in Eqs. (2-4) to the data and various subsets but apply this to the raw data rather than the binned frequencies and probabilities shown in Figs. 3 and 4. To do so we calculate the $\log$-likelihood $L$ according to

$L=\ln P_{\mathrm{D}}(A \mid \theta)=\sum_{i=1}^{n} \ln P_{\mathrm{D}}\left(A_{i} \mid \theta\right)$,

where $P_{\mathrm{D}}$ is a probability density model (e.g. Eqs. 2-4), and $\theta$ are the parameters to optimise. The log-likelihood of a particular set of parameters $\theta$ is therefore the sum of probabilities for all landslides in the data set or subset (of size $n$ ). Finding the combination of parameters that optimise $L$ gives the MLE of parameters for a given model.

To constrain the uncertainty on MLE parameters we perform bootstrap analyses in which we repeat the MLE method on 10000 data sets sampled by replacement from the SLI (and subsets). We therefore generate 10000 estimates of the most likely parameter combinations for the respective models and use the mean and standard deviation to report our most likely parameter combinations. For fitting of power-law distributions we use the MLE solutions for $\alpha$ provided by Clauset et al. (2009). Testing their solutions against our bootstrapping approach for fitting power-laws yields identical parameter estimates but with larger standard errors. We use these analytical error estimates for power law MLE, and report standard deviations about bootstrapped parameter means when performing MLE for double Pareto and inverse gamma functions (Eqs. 3 and 4, respectively).

\section{Results}

\subsection{Statistical distribution of landslide size in the UK}

The size-frequency distribution of the SLI is shown in Fig. 3. The probability distribution of landslides increases with landslide area, peaking at $1.0-7.0 \times 10^{-3} \mathrm{~km}^{2}$, before appearing to diminish in a power-law fashion (Fig. 3a). Previously documented event-driven landslide inventories show similar humped probability distributions (Brardinoni and Church, 2004; Guzzetti et al., 2008; Malamud et al., 2004; Pelletier et al., 1997; Stark and Hovius, 2001). A double Pareto distribution (Stark and Hovius, 2001) and a truncated inverse gamma function (Malamud et al., 2004) have also been plotted in Fig. 3a using MLE to find the best fit parameters. These 


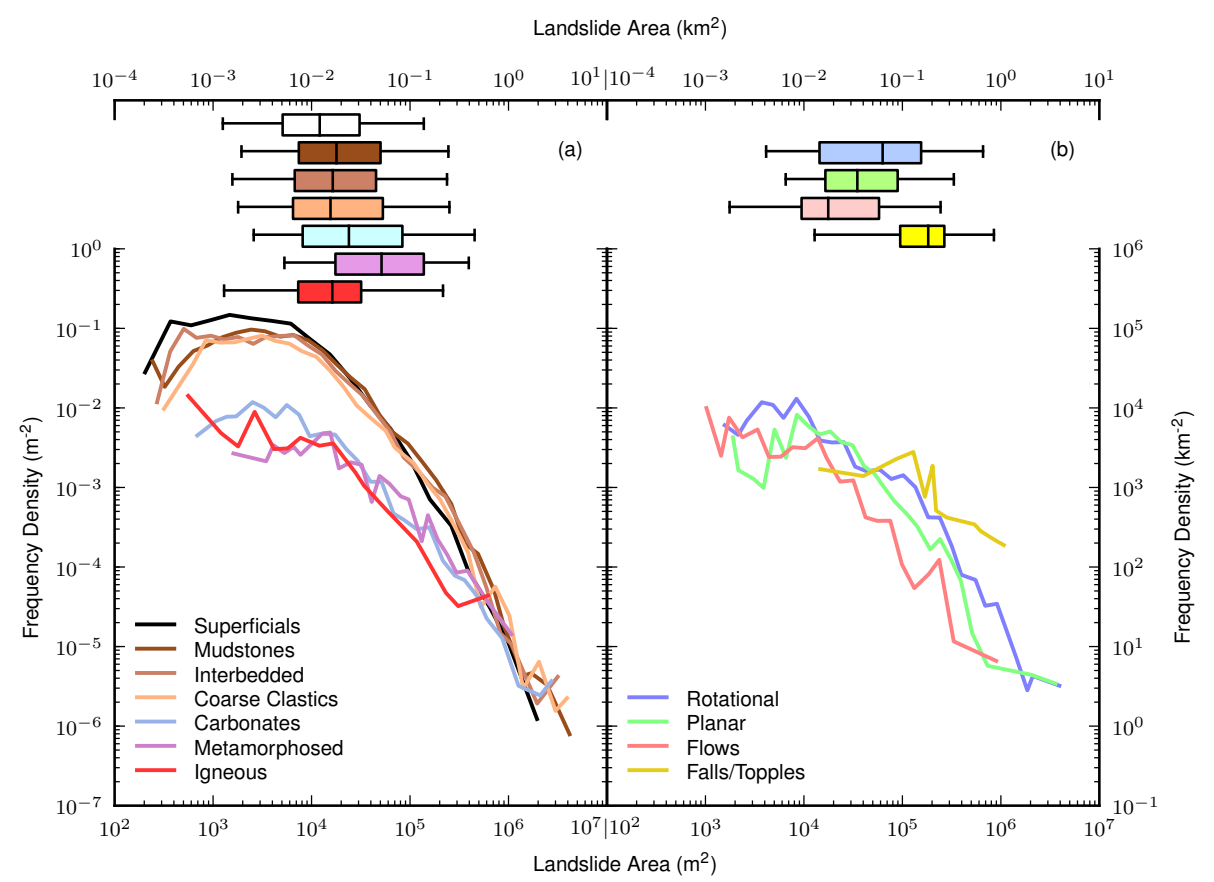

Figure 4. (a) Frequency distributions classified into broad lithologic groups for bins spaced evenly in logarithmic space. Table 1 provides details of the number of landslides in each group and the MLE parameter estimate for $\alpha$ for an assumed power-law distribution of mediumlarge landslides. Box plots show the median and lower/upper quartile statistics of area data for each lithologic group (whiskers are 5th and 95th percentiles). (b) Frequency distributions classified by type of mass movement process; rotational slides, planar slides, flows and falls/topples. Box plots show the median and lower/upper quartile statistics of area data for each lithologic group (whiskers are 5th and 95th percentiles).

functions coincide well with the observed probability distribution for UK landslides at areas $<10^{0} \mathrm{~km}^{2}$, although there is discrepancy between the data and model distributions for the largest mapped deposits. The median landslide size is $1.53 \times 10^{-2} \mathrm{~km}^{2}$ and the most frequent landslides are of the order $10^{-3}-10^{-2} \mathrm{~km}^{2}$. Figure $3 \mathrm{a}$ also shows the general distribution model postulated by Malamud et al. (2004) and attributed to complete landslide inventories associated with a trigger event (e.g. earthquake or storm). The peak probability in landslide size in the SLI is offset by roughly an order of magnitude compared to the general model, indicating fewer small landslides $\left(<10^{-2} \mathrm{~km}^{2}\right)$ in the SLI compared to complete event-driven landslide inventories. The general model of Malamud et al. (2004) is able to produce a reasonable fit for the frequency distribution of the largest landslides in the data set $\left(>10^{0} \mathrm{~km}^{2}\right)$ with $N_{\mathrm{T}}=10^{6}$ (Fig. 3b). The implications of this alternative fit are discussed in Sect. 4.

\subsection{Statistical distribution grouped by lithology and landslide type}

We subdivided the SLI into broad lithologic groups (Fig. 4a) sampled from BGS 1 : 50000 scale geological maps (British Geological Survey, 2010). The majority of landslides occur in superficial material, or clastic sedimentary rocks, particularly fine grained clays and muds and fines interbedded with coarser units. Based on the abundance of landslides (Table 1) and the distribution in Fig. 4a we refer to these as less-resistant lithologies. Landslides in carbonates, metamorphic and igneous units make up a relatively small part of the data set (see Table 1) and we refer to these as more resistant lithologies. Small landslides $\left(<10^{-2} \mathrm{~km}^{2}\right)$ are most abundant in superficial deposits, but medium-large landslides are more common in clastic sedimentary bedrock.

For more resistant lithologic groups there are similar numbers of large landslides $\left(\sim 10^{0} \mathrm{~km}^{2}\right)$ as there are in clastic sedimentary rocks, but smaller landslides are relatively infrequent (Fig. 4a). We quantified these observations by fitting a power-law relationship of the form of Eq. (2) by lithology fixing $A_{\min }=10^{-2} \mathrm{~km}^{2}$. Table 1 shows the MLE $\alpha$ determined following Clauset et al. (2009). Whilst a power law might not be the optimal distribution for every data set, we assume a power law in order to quantify an equivalent $\alpha$ for comparison. Our data indicate that landslides in more resistant lithologies may have lower exponents and therefore a proportionately greater number of large landslide events.

Finally, a subset of the SLI was plotted where information about the type of mass movement process was available $(n=$ $854)$. Figure $4 \mathrm{~b}$ shows the probability distribution for the four most common types of landslide; rotational slides $(n=373)$, planar slides $(n=303)$, flows $(n=131)$, and falls/topples 
$(n=47)$. Despite a much smaller sample size, these categories still broadly display rollover-power-law scaling for the landslide size-frequency relationship. The median event size decreases from rotational slides $\left(A=0.058 \mathrm{~km}^{2}\right)$ to planar slides $\left(A=0.033 \mathrm{~km}^{2}\right)$ and down to flow events $(A=$ $\left.0.021 \mathrm{~km}^{2}\right)$. This is not unexpected as rotational landslides tend to be large, deep-seated events involving significant amounts of bedrock, whilst flows tend to be hydraulically driven and mobilise material at the near-surface.

\section{Discussion}

Whilst we do not present a complete data set of all landslide occurrences in the UK, several features emerge from the results that speak to difference and similarities between eventdriven and secular landslide inventories, and to the important part that geology plays, each of which have implications for landslide hazard management.

\subsection{Landscape annealing and the small-landslide rollover}

Comparison of the SLI magnitude-frequency relationship (Fig. 3) to the proposed, general distribution for eventtriggered landslides (Malamud et al., 2004; Fig. 3a, grey line) reveals an order of magnitude offset between the peak areas. We interpret this to indicate the relative incompleteness of the SLI due to under-representation of small landslides. The causes behind this could include differences in the methods and coverage of landslide mapping resulting in the underrepresentation of small landslides, or difficulties in recognising small events in the field due to recolonisation by vegetation or subsequent erosion and redistribution of the deposit. We note that the NLD is not a complete landslide inventory and is constantly growing with the addition of newly observed historic and new landslides (Foster et al., 2012). Mapping of landslide deposits as part of the geological mapping program at the BGS is a continuing process and it is not expected that a complete coverage of landslide deposits in the UK has yet been achieved. This is demonstrated by a number of events in the NLD that did not link to an associated mapped deposit (Fig. 2) and hence were not incorporated into the SLI.

To test the extent to which small landslides are underrepresented in the SLI, we analysed separately a subset of the landslide data recently mapped in the North Yorkshire Moors, which are considered to be a substantially complete historic inventory (a comprehensive record of landslide deposits mapped through analysis of high resolution topography, aerial photography and field mapping). The area at which peak probability is observed is only slightly offset between the North Yorkshire data set $\left(2660 \mathrm{~m}^{2}\right)$ and the SLI $\left(3100 \mathrm{~m}^{2}\right)$, compared to the order of magnitude offset observed in relation to the general distribution proposed by Malamud et al. (2004) (Fig. 5). We suggest this slight dif-

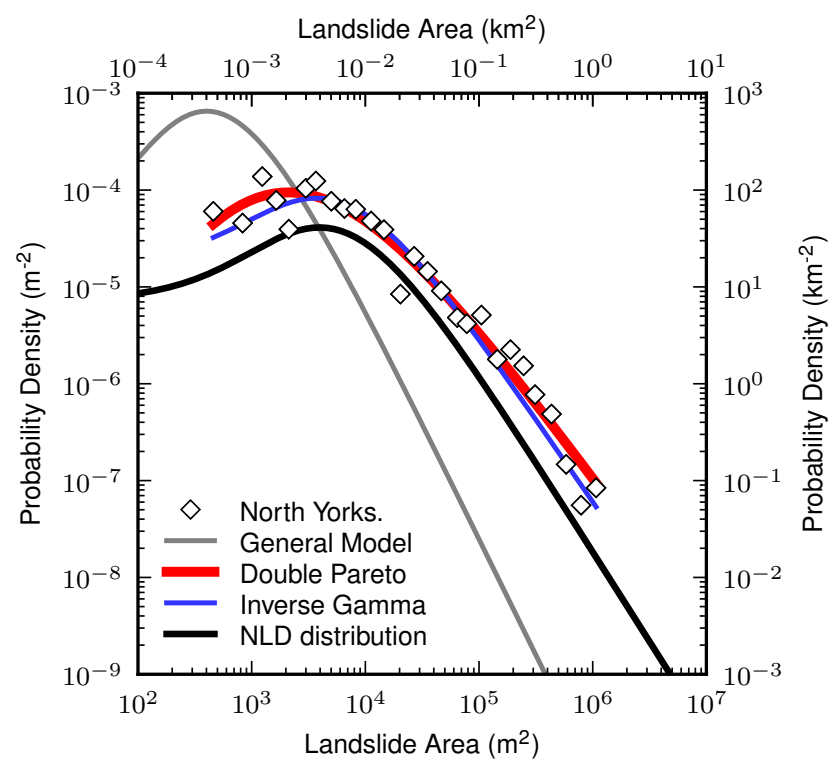

Figure 5. Probability distribution of landslide deposit area for landslides in North Yorkshire organised into logarithmically spaced bins (open diamonds). Solid black and grey lines show maximum likelihood estimates of a double Pareto function $\left(\alpha_{\mathrm{p}}=0.61\right.$; $\left.\beta=3.7 ; A_{\text {peak }}=927 \mathrm{~m}^{2}\right)$ and inverse gamma function $\left(\alpha_{g}=0.71\right.$; $r=9.57 \times 10^{-3} \mathrm{~km}^{2} ; s=-1.83 \times 10^{-3} \mathrm{~km}^{2}$ ) respectively, dashed grey and black lines show the general distribution proposed by Malamud et al. (2004) and the UK distribution from Fig. 3a, respectively. Note the similarity in shape between the North Yorkshire data set and the UK NLD fit (the vertical offset in probability density, is due to a difference in the range of landslide sizes considered and does not indicate relative probability).

ference may relate to the completeness of these data sets, with the likelihood that there are some smaller events in the NLD that were omitted when compiling the SLI. We stress, however, that there remains a large offset between the SLI and the general event based model proposed by Malamud et al. (2004), suggesting that this offset is real and likely the result of landscape annealing due to the loss of evidence of small events from the landscape.

\subsection{Role of lithology}

The frequency distributions for soft lithologies appear slightly curved in log-log space (Fig. 4a) suggesting that a power-law distribution may not be the most appropriate fit to the data. However, assuming a power-law model allows us to make comparisons between most likely parameters for different lithologic groups. Landslides in superficial deposits and soft lithologies dominate the SLI, whilst harder lithologic groups exhibit distinct magnitude-frequency scaling characterised by lower values of $\alpha$ setting a lower scaling gradient in log-log space (Fig. 4a; Table 1). This result has important implications for landslide size and associated hazard. Whilst there is significantly lower probability of small 
landslides in more resistant lithologies, the difference is minimal for larger landslides $\left(\sim 10^{6} \mathrm{~m}^{2}\right)$. Perhaps unsurprisingly the largest proportion of landslides and in particular smaller landslides $\left(<10^{3} \mathrm{~m}^{2}\right)$ occurs in poorly consolidated superficial deposits and hence characterisation of superficial materials will be important to site-based investigation of landslide susceptibility. Frattini and Crosta (2013) predict that size distributions should differ with material properties, such that weaker materials should result in more small, shallow landslides whilst stronger materials may promote relatively more large, deep seated landslides. Our findings also suggest that geology will play an important role in setting the size distribution of landslides, suggesting that the influence of lithology should be further explored.

Magnitude-frequency scaling of landslides classified by the type of mass movement have power-law scaling exponents $(\alpha=-1.3$ to -1.7$)$ lower than the data set as a whole $(\alpha=-1.71)$. Lower exponents suggests the subset of data may be biased towards larger events, and indeed it seems likely (and reasonable) that detailed field studies to determine the style of failure are preferentially carried out for larger failure events. Unfortunately there are few observations of landslide type below areas of $10^{3} \mathrm{~m}^{2}$ (Fig. 4b) yet there are a large number of landslides in the NLD of this magnitude (Fig. 3b). However, whilst the sample sizes are small, there is a suggestion that the gradient of the most likely power law decreases with landslide type. This would be broadly consistent with the results of Brunetti et al. (2009) who performed analysis of landslide volume distributions rather than area as in the present study. Brunetti et al. (2009) performed magnitude-frequency analysis of 19 landslide inventories and found that the scaling exponents for landslide volume were lower for rock falls and rock slides than for slides and soil slides. More data are needed to provide an empirical test that more resistant lithologies preferentially yield deep-seated landslides whilst weaker materials preferentially yield shallow landslides, as found theoretically by Frattini and Crosta (2013).

\subsection{The large-landslide deficit}

A national landslide inventory for Italy comprising 377000 landslides (Trigila et al., 2010) exhibits power-law scaling above $10^{-2} \mathrm{~km}^{2}$ similar to the SLI (Fig. 3a). Interestingly both data sets show deviation from fitted scaling relationships for the largest landslides $\left(>10^{0} \mathrm{~km}^{2}\right.$ for the UK; $>10^{1} \mathrm{~km}^{2}$ in Italy) suggesting that either we are undersampling with respect to the largest landslides or large events are less frequent than power-law scaling would predict. The difference in cutoff areas between the two data sets may be the result of only reporting the areas of mapped deposits in the UK whilst in Trigila et al. (2010) area refers to the combined source and sink outline.

There are a number of possible explanations for this apparent deficit of large landslides. Firstly, the data set is expected to be incomplete and there may be some large landslides that have not been recorded. It seems unlikely, however, that the deficit represents observational bias, since large landslides should be the most prominent in the landscape. The deficit may, in fact, be larger, as inspection shows that some of the largest mapped deposit areas consist of amalgamated deposits of numerous smaller events. Therefore, we suggest the deficit is real. Possible explanations for the deficit aside from an incomplete database, which we describe below, include an incomplete landscape, and/or a temporal transience in the occurrence of landslides since the last glacial maximum (LGM).

For a large landslide to occur requires a large slope. The availability of the highest relief is spatially limited in the UK to central and northern Wales, the Lake District and the Scottish Highlands. As previously observed, data coverage in these regions is not as extensive as in other parts of the British Isles. The relative paucity of large slopes (and associated large landslides) elsewhere in the country may result in the large-landslide deficit. We refer to this as an incomplete landscape. Figure 6 shows the spatial density of mapped landslides comprising the SLI. Whilst we would anticipate that areas with the greatest relief and steepest hillslope gradients might contain the highest density of landslides, this seems not to be the case in the SLI. Coverage of landslide mapping in the Scottish Highlands and parts of northern Wales are sparser than other low relief areas of the UK. This is of particular relevance since these areas will have large slopes which may yield large landslides, and these areas tend to be underlain by more resistant lithologies.

It is likely that the bulk of landslides range in age from the LGM ( $27 \mathrm{ka}$; Clark et al., 2012) to the present day. During this time, climate will have varied as the British Ice Sheet receded, and mass movement processes are likely to have been initially more active as soils and regolith both warmed and lost structural support from ice cover and permafrost. We speculate, therefore, that many landslides and certainly most of the larger landslides would occur early in this LGM-topresent time span, during the paraglacial transition (Ballantyne, 2002; Dadson and Church, 2005). Unlike active mountain belts, steep slopes will not be regenerated by continued rock uplift and erosion, and therefore the drivers for those landslides are gradually reduced over time as the emerging landscape passes through a period of readjustment to new and more stable conditions. Instability likely continued through the variable climate immediately prior to the Holocene, and returned again during the latter part of the Holocene (Neolithic times, in particular) as extensive anthropogenic forest clearance and land-use changes occurred. These latter processes, all else being equal, would lead to an increase in the rate of landslide activity, consistent with rapid Neolithic valley sedimentation observed in many parts of the UK (Brown, 2009). We suggest, therefore, that the population of landslides in the SLI may be dominated by the relatively rapid denudation of early post-LGM and early anthropogenic times. 


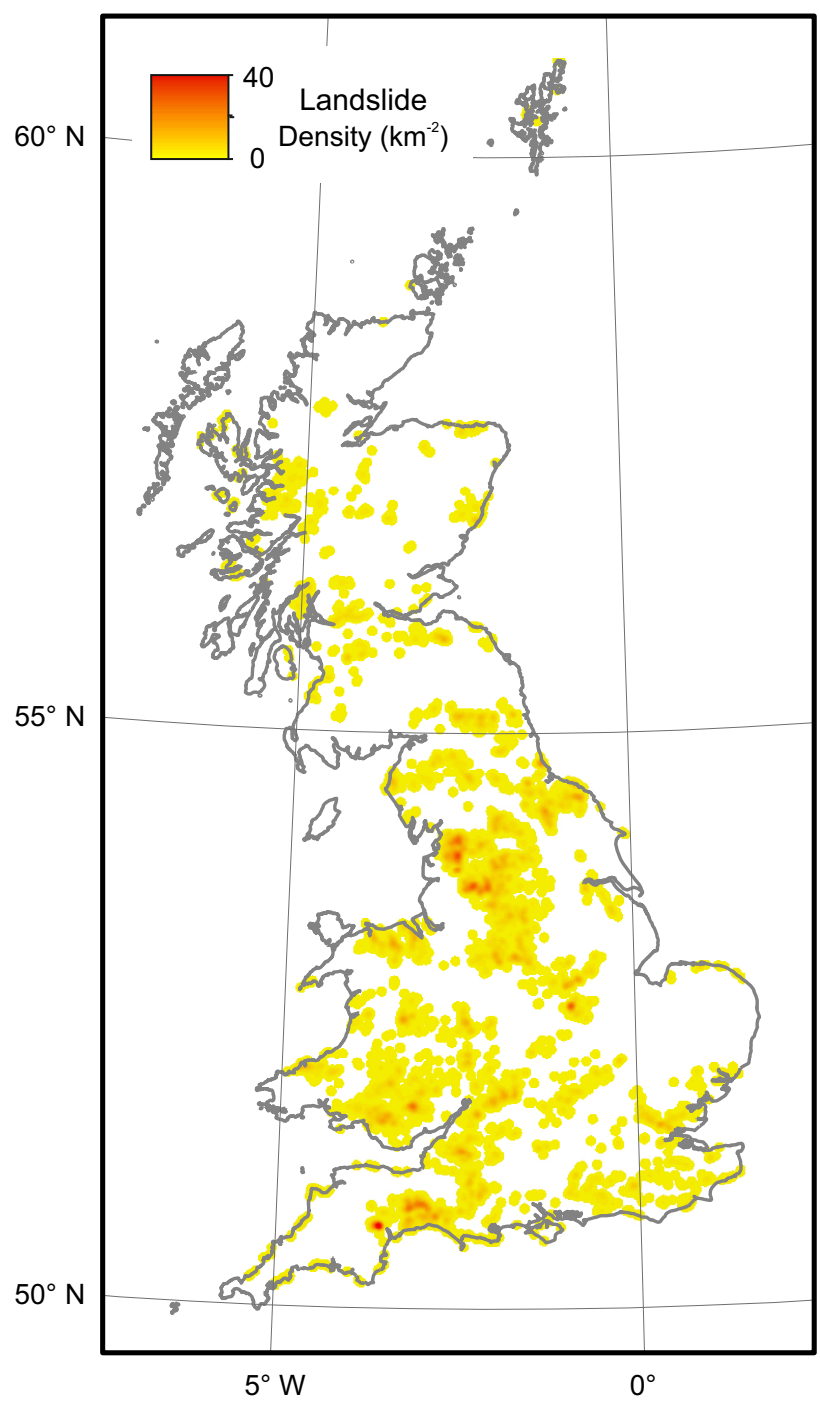

Figure 6. Map of landslide density (number of landslides per $\mathrm{km}^{2}$ ) across the UK derived from mapped landslide deposits. Data is gridded to $1 \mathrm{~km}$ and calculated using a $5 \mathrm{~km}$ search radius. Low landslide densities in Scotland and NE Wakes may indicate that coverage is particularly poor.

As a result, relatively large landslides show a deficit with respect to a model fit that is derived principally from the relatively greater number of smaller- to moderate-sized landslides.

An alternative perspective is provided by the areafrequency analysis (Fig. 3b), which would suggest that largelandslide deficit is only apparent, and that it is smaller- and moderate-sized landslides that are in deficit. To reach this conclusion would require the assumption that the general model proposed by Malamud et al. (2004) was appropriate to represent the probability density for all landslides in the UK since the LGM. Moreover, it would suggest that the landscape annealing processes by which small events are lost from the geomorphic record not only act to offset the posi- tion of the "hump" in historic landslide inventories, but also reduce the exponent $\alpha$ through time. Data for historic inventories presented by Malamud et al. (2004) (after Guzzetti et al., 2003; Ohmori and Sugai, 1995) suggest that this is not the case because $\alpha$ appears to be conserved in those historic inventories (see also Guzzetti et al., 2008; Trigila et al., 2010). Thus it remains unclear whether Malamud's general model is appropriate for a secular landslide inventory spanning several thousand years and a highly variable external forcing, during which time there is reason to suspect variation in the frequency (and possibly size) of landslides.

\subsection{Implications for landslide hazards}

The combined results here have implications for the assessment of landslide hazards and ultimately for landslide risk management. At face value, for example, the modelfits (double Pareto or inverse gamma; see Fig. 3) presented here yield a low frequency of small landslides relative to complete, event-triggered landslide databases. This size category includes anything from 3 to $30 \mathrm{~m}$ in equivalent radius, which can be hazardous to a wide variety of infrastructure. It is more likely, however, that the SLI significantly underrepresents landslides of this size, as we argue above. In other words, the national-scale, small-landslide hazard is greater than the SLI would suggest (Fig. 3a; Table 1). Our results also tell us that the occurrence of landslides of any particular size is largely independent of type (i.e. scaling between size and frequency still follows a power law with rollover), but that type and magnitude are linked, with deep-seated rotational landslides tending to be larger than planar slides and flows. The role of lithology emerges as control by two broad classes of bedrock, (resistant: carbonates, metamorphic and igneous rocks vs. less resistant: superficial, mudstones, interbedded, and coarse clastics) each characterised by a distinct power-law distribution and each (with the exception of igneous rocks) showing a rollover at relatively small landslides. We also suggest that the discrepancy between model and observations for relatively large landslides may be a function of a transient landslide response as the UK emerged from glacial conditions and into an initially variable (e.g. Allerød warming and Younger Dryas events) then relatively stable Holocene climate. In other words, the national-scale large-landslide hazard is lower than predicted by the model fit to the SLI (Fig. 3a and Table 1). It is important to emphasise that the SLI is a sample of the NLD (itself an incomplete record of all past landslides in the UK), and that it does not include coastal landslides. Importantly, the proposed process of annealing and the potentially transient response of hillslopes have the consequence that it is not possible to use the statistical properties of the current SLI database to rigorously constrain probabilities of future landslides in the UK. 


\section{Conclusions}

Analysis of a national (UK) secular landslide inventory reveals a statistical distribution that can be well characterised by an inverse gamma or double Pareto distribution with a well-defined rollover at a landslide area between 10 and $30 \mathrm{~m}^{2}$. The power-law component for medium-large landslides has a scaling exponent $\alpha=-1.71 \pm 0.02$. This general form of the distribution is similar to that found for many single-event driven landslides, although there are two important specific differences. First: the magnitude of the smalllandslide rollover occurs at a significantly larger size than in single-event samples. We interpret this as a reflection of landscape annealing processes (e.g. recolonisation by vegetation, reworking of landslide deposits), with the corollary that the model fit would underestimate the frequency of relatively small landslides. Second: we observe a deficit, relative to the model fit, in the largest landslides. Possible explanations for this deficit include (i) poor data coverage in areas where large landslides might be expected; (ii) spatial limitation in the occurrence of large landslides due to lack or limited occurrence of large hillslopes; and (iii) temporal transience or non-linear response of the UK landscape as it emerged from the LGM; and, during the Neolithic, accelerated landscape change due to human activity. In such a scenario, most of the landslides, certainly the larger ones, are likely to have formed early in the post-LGM time span as the soil-regolith-bedrock column lost support of both ice and/or permafrost.

Acknowledgements. We thank Claire Dashwood, Catherine Pennington, Vanessa Banks, Helen Reeves and Bruce Malamud for discussions that greatly improved the sampling techniques and interpretations presented here. Thanks also to Ken Lawrie for assistance with the NLD, to Murray Lark and Ben Marchant for guidance on statistical analyses, and the Associate Editor, Dimitri Lague, and two anonymous reviewers for the many suggestions that improved the final paper. This paper is published with the permission of the Executive Director of the British Geological Survey and was supported in part by the Climate and Landscape Change research programme at the BGS.

Edited by: D. Lague

\section{References}

Ballantyne, C. K.: Paraglacial geomorphology, Quaternary Sci. Rev., 21, 1935-2017, 2002.

Brardinoni, F. and Church, M.: Representing the landslide magnitude-frequency relation: Capilano River basin, British Columbia, Earth Surf. Proc. Land., 29, 115-124, 2004.

British Geological Survey: Digital Geological Map of Great Britain 1:10000 scale (DiGMapGB-10) data. Version 2.18. Keyworth, Nottingham, British Geological Survey, Release date: 15 January 2009, 2009.

British Geological Survey: Digital Geological Map of Great Britain 1:50000 scale (DiGMapGB-50) data. Version 6.20. Keyworth,
Nottingham, British Geological Survey, Release date: 14 October 2010, 2010.

Brown, A. G.: Colluvial and alluvial response to land use change in Midland England: An integrated geoarchaeological approach, Geomorphology, 108, 92-106, 2009.

Brunetti, M. T., Guzzetti, F., and Rossi, M.: Probability distributions of landslide volumes, Nonlin. Processes Geophys., 16, 179-188, doi:10.5194/npg-16-179-2009, 2009.

Clark, C. D., Hughes, A. L. C., Greenwood, S. L., Jordan, C., and Sejrup, H. P.: Pattern and timing of retreat of the last British-Irish Ice Sheet, Quaternary Sci. Rev., 44, 112-146, 2012.

Clarke, B. A. and Burbank, D. W.: Bedrock fracturing, threshold hillslopes, and limits to the magnitude of bedrock landslides, Earth Planet. Sc. Lett., 297, 577-586, 2010.

Clauset, A., Shalizi, C. R., and Newman, M. E.: Power-law distributions in empirical data, SIAM review, 51, 661-703, 2009.

Crozier, M.: Deciphering the effect of climate change on landslide activity: A review, Geomorphology, 124, 260-267, 2010.

Dadson, S. J. and Church, M.: Postglacial topographic evolution of glaciated valleys: a stochastic landscape evolution model, Earth Surf. Proc. Land., 30, 1387-1403, 2005.

Foster, C., Pennington, C. V. L., Culshaw, M., and Lawrie, K.: The national landslide database of Great Britain: development, evolution and applications, Environ. Earth Sci., 66, 941-953, doi:10.1007/s12665-011-1304-5, 2012.

Frattini, P. and Crosta, G. B.: The role of material properties and landscape morphology on landslide size distributions, Earth Planet. Sc. Lett., 361, 310-319, 2013.

Galli, M., Ardizzone, F., Cardinali, M., Guzzetti, F., and Reichenbach, P.: Comparing landslide inventory maps, Geomorphology, 94, 268-289, 2008.

Gibson, A. D., Culshaw, M. G., Dashwood, C., and Pennington, C. V. L.: Landslide management in the UK-the problem of managing hazards in a "low-risk" environment, Landslides, 10, 599610, 2013.

Glade, T.: Landslide occurrence as a response to land use change: a review of evidence from New Zealand, CATENA, 51, 297-314, 2003.

Guzzetti, F., Malamud, B. D., Turcotte, D. L., and Reichenbach, P.: Power-law correlations of landslide areas in central Italy, Earth Planet. Sc. Lett., 195, 169-183, 2002.

Guzzetti, F., Reichenbach, P., Cardinali, M., Ardizzone, F., and Galli, M.: The impact of landslides in the Umbria region, central Italy, Nat. Hazards Earth Syst. Sci., 3, 469-486, doi:10.5194/nhess-3-469-2003, 2003.

Guzzetti, F., Ardizzone, F., Cardinali, M., Galli, M., Reichenbach, P., and Rossi, M.: Distribution of landslides in the Upper Tiber River basin, central Italy, Geomorphology, 96, 105-122, 2008.

Guzzetti, F., Mondini, A. C., Cardinali, M., Fiorucci, F., Santangelo, M., and Chang, K. T.: Landslide inventory maps: New tools for an old problem, Earth-Sci. Rev., 112, 42-66, 2012.

Hovius, N., Stark, C. P., and Allen, P. A.: Sediment flux from a mountain belt derived by landslide mapping, Geology, 25, 231234, 1997.

Jones, D. K. C. and Lee, E. M.: Landsliding in Great Britain, Department of the Environment, London, 1994.

Keiler, M., Knight, J., and Harrison, S.: Climate change and geomorphological hazards in the eastern European Alps, Philos. T. R. Soc. A, 368, 2461-2479, 2010. 
Korup, O., Densmore, A. L., and Schlunegger, F.: The role of landslides in mountain range evolution, Geomorphology, 120, 77-90, 2010.

Korup, O., Görüm, T., and Hayakawa, Y.: Without power? Landslide inventories in the face of climate change, Earth Surf. Proc. Land., 37, 92-99, 2012.

Larsen, I. J., Montgomery, D. R., and Korup, O.: Landslide erosion controlled by hillslope material, Nat. Geosci., 3, 247-251, 2010.

Malamud, B. D., Turcotte, D. L., Guzzetti, F., and Reichenbach, P.: Landslide inventories and their statistical properties, Earth Surf. Proc. Land., 29, 687-711, 2004.

Ohmori, H. and Sugai, T.: Toward geomorphometric models for estimating landslide dynamics and forecasting landslide occurrence in Japanese mountains, Z. Geomorphol. Supp., 101, 149164, 1995.

Parker, R. N., Densmore, A. L., Rosser, N. J., De Michele, M., Li, Y., Huang, R., Whadcoat, S., and Petley, D. N.: Mass wasting triggered by the 2008 Wenchuan earthquake is greater than orogenic growth, Nat. Geosci., 4, 449-452, 2011.

Pelletier, J. D., Malamud, B. D., Blodgett, T., and Turcotte, D. L.: Scale-invariance of soil moisture variability and its implications for the frequency-size distribution of landslides, Eng. Geol., 48, 255-268, 1997.

Petley, D.: Global patterns of loss of life from landslides, Geology, 40, 927-930, 2012.

Schuster, R. L.: Socioeconomic Significance of Landslides, Landslides: Investigation and Mitigation, National Academies Press, Transportation Research Board Special Report, 12-35, 1996.
Stark, C. P. and Guzzetti, F.: Landslide rupture and the probability distribution of mobilized debris volumes, J. Geophys. Res.-Earth, 114, F00A02, doi:10.1029/2008JF001008, 2009.

Stark, C. P. and Hovius, N.: The characterization of landslide size distributions, Geophys. Res. Lett., 28, 1091-1094, 2001.

Styles, M. T., Gillespie, M. R., Bauer, W., and Lott, G. K.: Current status and future development of the BGS Rock Classification Scheme, British Geological Survey, Nottingham, UK, 2006.

Trigila, A., Iadanza, C., and Spizzichino, D.: Quality assessment of the Italian Landslide Inventory using GIS processing, Landslides, 7, 455-470, 2010.

Turcotte, D. L., Malamud, B. D., Guzzetti, F., and Reichenbach, P.: Self-organization, the cascade model, and natural hazards, P. Natl. Acad. Sci. USA, 99(Suppl. 1), 2530-2537, 2002.

Van Den Eeckhaut, M. and Hervás, J.: State of the art of national landslide databases in Europe and their potential for assessing landslide susceptibility, hazard and risk, Geomorphology, 139140, 545-558, 2012.

Van Den Eeckhaut, M., Poesen, J., Govers, G., Verstraeten, G., and Demoulin, A.: Characteristics of the size distribution of recent and historical landslides in a populated hilly region, Earth Planet. Sc. Lett., 256, 588-603, 2007.

Walsby, J. C.: GeoSure; a bridge between geology and decisionmakers, Geological Society, London, Special Publications, 305 , 81-87, 2008.

Winter, M., Dent, J., Macgregor, F., Dempsey, P., Motion, A., and Shackman, L.: Debris flow, rainfall and climate change in Scotland, Q. J. Eng. Geol. Hydroge., 43, 429-446, 2010. 